\title{
Olanzapine for the treatment of borderline personality disorder: variable dose 12-week randomised double-blind placebo-controlled study
}

\author{
S. Charles Schulz, Mary C. Zanarini, Anthony Bateman, Martin Bohus, Holland C. Detke, \\ Quynh Trzaskoma, Yoko Tanaka, Daniel Lin, Walter Deberdt and Sara Corya
}

\section{Background}

Despite the prevalence and clinical significance of borderline personality disorder, its treatment remains understudied.

\section{Aims \\ To evaluate treatment with variably dosed olanzapine in individuals with borderline personality disorder. \\ Method \\ In this 12-week randomised, double-blind trial, individuals received olanzapine $(2.5-20 \mathrm{mg} /$ day; $n=155)$ or placebo $(n=159)$ (trial registry: NCT00091650). The primary efficacy measure was baseline to end-point change on the Zanarini Rating Scale for Borderline Personality Disorder (ZAN-BPD) using last-observation-carried-forward methodology.}

\section{Results}

Both olanzapine and placebo groups showed significant improvements but did not differ in magnitude at end-point $(-6.56 \mathrm{~V} .-6.25, P=0.661)$. Response rates $(50 \%$ reduction in ZAN-BPD) were $64.7 \%$ with olanzapine and $53.5 \%$ with placebo $(P=0.062)$; however, time to response was significantly shorter for olanzapine $(P=0.022)$. Weight gain was significantly greater $(2.86 \mathrm{~V} .-0.35 \mathrm{~kg}, P<0.001)$, with higher incidence of treatment-emergent abnormal high levels of prolactin for the olanzapine group.

\section{Conclusions}

Individuals treated with olanzapine and placebo showed significant but not statistically different improvements on overall symptoms of borderline personality disorder. The types of adverse events observed with olanzapine treatment appeared similar to those observed previously in adult populations.

\section{Declaration of interest}

This study was sponsored by Eli Lilly. S.C.S. has received honorarium from Eli Lilly, AstraZeneca and Bristol-Meyers Squibb; grant fees from Eli Lilly, AstraZeneca, Abbott, MIND Institute and the $\mathrm{NIMH}$; and consultation fees from Eli Lilly, AstraZeneca and Vanda. H.C.D., Q.T., Y.T., D.L. and S.C. are employed by Lilly Research Laboratories.
Borderline personality disorder is a serious psychiatric illness that affects approximately $1-2 \%$ of the adult population during their lifetime, and approximately $20 \%$ of psychiatric in-patients. ${ }^{1,2}$ People with the disorder suffer from severe psychosocial dysfunction $^{3}$ and the mortality rate due to suicide can approach $10 \%$. Despite the prevalence and clinical significance of borderline personality disorder, its treatment remains understudied. Although a number of smaller studies have shown some benefits of treatment with antipsychotic drugs, ${ }^{5-12}$ no medication has been approved for the treatment of borderline personality disorder. The objective of the present study was to assess the efficacy and safety of variably dosed olanzapine in people with borderline personality disorder in a large double-blind placebo-controlled trial.

\section{Method}

This was one of two large, multicentre, concurrent, parallel, double-blind randomised placebo-controlled clinical trials comparing olanzapine with placebo in people with borderline personality disorder (trial registry: NCT00091650). The second study was a placebo-controlled dose comparison study and is presented elsewhere. $^{13}$

The present study was conducted at 52 sites in nine countries (Belgium, France, Germany, Norway, Portugal, Spain, Sweden, UK and USA). Appropriate ethics review boards approved the study before recruitment. All participants received a thorough explanation of the study protocol and written informed consent was obtained prior to participation in the study.

\section{Study design}

The study consisted of a 2-14 day screening period followed by a 12-week double-blind acute treatment period. Participants who completed the 12-week double-blind period were eligible to enter a 12-week open-label extension phase. This report presents results through the 12-week double-blind acute treatment period.

The 12-week duration of the double-blind treatment period of this study was considered sufficient to confirm a sustained difference between treatment groups, surpassing temporary variability in condition. Furthermore, participant drop-out rates in previous studies of borderline personality disorder exceeded $50 \%$ after 12 weeks of therapy. ${ }^{11,14,15}$ Individuals who met enrolment criteria were randomised in a 1:1 ratio to receive treatment with olanzapine $(2.5-20 \mathrm{mg} /$ day $)$ or placebo. All participants, study site personnel and investigators were masked to randomisation codes.

For individuals assigned to olanzapine treatment, the starting dosage was 2.5 or $5 \mathrm{mg} /$ day according to the investigator's judgement. After 1 week, the dose could be increased in 2.5 or $5 \mathrm{mg}$ increments up to a maximum of $20 \mathrm{mg} /$ day and could be decreased as needed per the investigator's judgement. To encourage sufficient dosing, beginning at week 4, participants not demonstrating a $\geqslant 30 \%$ decrease from their baseline total score on the Zanarini Rating Scale for Borderline Personality Disorder $(\mathrm{SAN}-\mathrm{BPD})^{16}$ were prescribed dose increases in $2.5-$ $5 \mathrm{mg}$ increments within a range of $5-20 \mathrm{mg} / \mathrm{day}$ unless an adverse event precluded an increase in dose. Investigators were not required to calculate the criteria for dose increases. If an individual reached the maximum dose but failed to achieve a $\geqslant 30 \%$ reduction from baseline on the ZAN-BPD score, that 
person could remain in the study at the investigator's discretion. In the event that an investigator felt that the person's dose needed to be increased in between clinic visits, the investigator could do so by bringing them into the clinic to conduct an unscheduled visit.

\section{Participants}

Eligible participants were male or female out-patients 18-65 years of age who met all of the DSM-IV general diagnostic criteria for a personality disorder and DSM-IV criteria for borderline personality disorder as determined by the Diagnostic Interview for DSM-IV Personality Disorders (DIPD-IV). ${ }^{17}$ Participants had to have a ZAN-BPD total score of 9 at the time of randomisation. People were excluded from the study if they had ever met criteria for schizophrenia, schizoaffective disorder, schizophreniform disorder, bipolar I disorder, or delusional disorder as assessed by the Structured Clinical Interview for DSM-IV Axis I disorders. ${ }^{18}$ Participants could not have a diagnosis of major depressive disorder, bipolar II disorder, or substance dependence within the previous 3 months; could not be actively suicidal; could not have a current diagnosis of posttraumatic stress disorder, panic disorder, or obsessive-compulsive disorder; could not have a body mass index less than 17; and could not meet criteria for a cluster A personality disorder. These restrictive diagnostic criteria were necessary in order to evaluate the effect of olanzapine specifically on borderline personality disorder. Although many of the excluded comorbidities are common among people with borderline personality disorder, inclusion of those comorbidities could obfuscate the results of the study due to potential treatment effects on comorbid symptoms rather than direct effects on borderline personality disorder symptoms.

Concomitant use of benzodiazepines/hypnotics was allowed during the study at a dose of no more than $1 \mathrm{mg}$ lorazepam equivalents/day. Episodic use of anticholinergics was permitted at a dose up to $6 \mathrm{mg} /$ day benzatropine mesilate or biperiden (an antiparkinsonian agent), or up to $12.0 \mathrm{mg}$ /day trihexyphenidyl, to treat extrapyramidal symptoms (EPS); however, the use of anticholinergic medication as prophylaxis for EPS was not allowed. Use of other psychotropic drugs was not permitted and all such medications were discontinued prior to randomisation. Participants could not be on antidepressant, mood stabiliser, or antipsychotic medication within 1 week of randomisation (within 4 weeks for fluoxetine, and within 1 injection interval for depot antipsychotics).

To avoid possible confounds owing to the use of psychotherapy, people entering the study could not begin any type of psychotherapy within 3 months prior to enrolment or during the double-blind study period. Individuals were permitted to enter the study on psychotherapy if they had been receiving psychotherapy for $>3$ months at the time of enrolment, but they could not change their psyochotherapy regimen during the course of the study.

\section{Assessments}

Participants were assessed in the clinic weekly for the first 2 weeks of treatment and every other week thereafter (weeks 1, 2, 4, 6, 8, 10 and 12).

\section{Zanarini Rating Scale for Borderline Personality Disorder}

The primary efficacy variable was the last-observation-carriedforward (LOCF) mean change from baseline to end-point in ZAN-BPD total score. The ZAN-BPD consists of a semistructured interview with ratings from 0 (no symptoms) to 4 (severe symptoms) on each of nine items corresponding to the nine DSM-IV criteria for borderline personality disorder. Thus, ZAN-BPD total scores can range from 0 to 36 .

Secondary efficacy variables included mean baseline to endpoint changes on the ZAN-BPD item scores as well as on the following measures.

\section{Symptom Checklist-90-Revised (SCL-90-R)}

The SCL-90- $\mathrm{R}^{19}$ is a 90 -item, self-administered questionnaire to measure the outcome or status of psychopathology and to quantify current psychopathology along nine symptom constructs: somatisation, obsessive-compulsive symptoms, interpersonal sensitivity, depression, anxiety, anger-hostility, phobic-anxiety, paranoid ideation, and psychoticism. A Global Severity Index (GSI) can be calculated as a total of all (completed) items and the domain constructs may be scored individually. The GSI and dimension scores were analysed.

\section{Global Assessment of Functioning (GAF)}

The $\mathrm{GAF}^{20}$ is a clinician-rated, 100-point instrument indicating overall psychosocial functioning (psychological symptoms and social and occupational functioning) during a specified period on a continuum from psychological sickness to health. The summary score reflects the level of a individual's overall functioning.

\section{Sheehan Disability Scale}

The Sheehan Disability Scale ${ }^{21}$ is a self-rated instrument used to measure the effect of the individual's symptoms on three areas: work/school, social life and family life/home responsibilities. Each area is scored according to how much it was disrupted by symptoms $(0=$ not at all to $10=$ very severely $)$ and the total score ranged from 0 to 30 .

\section{Overt Aggression Scale-Modified (OAS-M)}

The OAS $-\mathrm{M}^{22}$ is a clinician-administered, semi-structured interview designed to assess various manifestations of aggressive behaviour in out-patients. It consists of three separate subscales: aggression, irritability and suicidality scores.

\section{Montgomery-Åsberg Depression Rating Scale (MADRS)}

The MADRS ${ }^{23,24}$ is a 10 -item clinician-rated measure of severity of depressive symptoms.

\section{Response}

Additional efficacy measures included rates of response (defined a priori using two criterion levels: $\geqslant 30 \%$ and $\geqslant 50 \%$ decrease in ZAN-BPD total score from baseline at any time) and time to response.

\section{Safety}

Safety was assessed by evaluating between-group differences for treatment-emergent adverse events and changes (mean and/or categorical) in vital signs, electrocardiogram (ECG) findings, laboratory values, or EPS from baseline to end-point or at any time. Electrocardiogram and laboratory measures were performed during the screening period and at the final visit. Extrapyramidal symptom evaluations were performed during the screening period, at weeks 2, 4, and 8, and at the final visit. Laboratory tests included clinical chemistry, electrolytes, lipid profile, prolactin, urinalysis, and hematology panels. These tests were performed 
at the protocol-specified time points, when clinically indicated, and any time a participant completed the double-blind acute period or discontinued the study. Criteria from the National Cholesterol Education Program ${ }^{25}$ guidelines were used for determining treatment-emergent changes in fasting total cholesterol $(<5.172 \mathrm{mmol} / \mathrm{l}$ at baseline to $\geqslant 6.206 \mathrm{mmol} / \mathrm{l}$ anytime postbaseline), low-density lipoprotein (LDL) cholesterol $(<2.586 \mathrm{mmol} / \mathrm{l}$ to $\geqslant 4.138 \mathrm{mmol} / \mathrm{l})$, high-density lipoprotein cholesterol $(\geqslant 1.034 \mathrm{mmol} / \mathrm{l}$ to $<1.034 \mathrm{mmol} / \mathrm{l})$ and triglycerides $(<1.694 \mathrm{mmol} / \mathrm{l}$ to $\geqslant 2.258 \mathrm{mmol} / \mathrm{l})$. Criteria from the American Diabetes Association ${ }^{26}$ were used to determine treatment-emergent changes in fasting glucose $(<6.994 \mathrm{mmol} / \mathrm{l}$ to $\geqslant 6.994 \mathrm{mmol} / \mathrm{l})$. Extrapyramidal symptoms were assessed using the Simpson-Angus Scale, ${ }^{27}$ the Barnes Akathisia Rating Scale, ${ }^{28}$ and the Abnormal Involuntary Movement Scale (AIMS). ${ }^{29}$ All adverse events were recorded as actual terms and coded to Medical Dictionary for Regulatory Activities (MedDRA) terms (www.meddramsso.com/ MSSOWeb/index.htm). Participant adherence with study medication was assessed at each visit by direct questioning and study drug accountability (pill count).

\section{Telephone calls}

During the weeks participants did not visit the clinic (weeks 3, 5, 7, 9 and 11), participants received telephone calls conducted by the principal investigator, sub-investigator or study coordinator. Telephone calls consisted of a brief discussion of the individual's general condition, followed by assessments of concomitant medication usage, study drug adherence, adverse events and nutrition and fitness, all of which were also assessed at the regular clinic visits. If the site personnel who called the participant was not a physician and determined that a dose decrease was necessary, or if the participant reported a serious adverse event, a study physician also called them.

\section{Statistical methods}

All participants' data were analysed, as stated in the protocol, on an intent-to-treat basis. Participant's characteristics at baseline, including demographics (gender, age and origin), illness characteristics and baseline scores on efficacy measures, were summarised for both treatment groups. Frequencies were compared using Fisher's exact test. Means were compared by
ANOVA with treatment and investigator as the independent factors for the continuous data.

For analysis of change from baseline during the treatment period, people with a baseline and at least 1 post-baseline measurement were included in the analysis. Changes in continuous efficacy data were analysed with analysis of covariance (ANCOVA) models, which included terms for the fixed effects of baseline, investigator and treatment. All reported ZAN-BPD, SCL-90-R, MADRS, OAS-M, Sheehan, and GAF mean change scores represent least squares means. Cohen effect size estimates were used when comparing baseline to end-point changes in ZAN-BPD scores between treatment groups. Analysis of visit-wise ZAN-BPD total scores used a mixed-effects model repeated measures (MMRM) method, which included independent factors for baseline, therapy, investigator, visit and therapy visit interaction, and which statistically controls for participant drop out over time. Frequency of treatment response was analysed using Fisher's exact test. Time to response was calculated using the Kaplan-Meier technique with treatment comparisons made using the log-rank test. The LOCF mean changes from baseline to endpoint for continuous safety measures were analysed using analysis of variance (ANOVA) models, including terms for the fixed effects of investigator and treatment. Categorical analyses of safety data were analysed using Fisher's exact test.

All tests of hypotheses were tested at a two-sided alpha level of 0.05 . We used SAS software version 8.2, using a multiple virtual system on an IBM mainframe computer (SAS Institute Inc, Cary, North Carolina, USA) to perform all statistical analyses.

\section{Results}

A total of 385 participants were screened for eligibility, and 314 participants were randomly assigned to receive olanzapine $(n=155)$ or placebo $(n=159)$. The trial flow diagram is shown in Fig. 1. The mean number of individuals per study site was 6.5 (s.d.=5.0) and ranged from 1 to 26. There were no statistically significant differences in baseline demographic or illness characteristics between the treatment groups (Table 1). Rates of discontinuation from the 12-week acute phase were $48.4 \%$ (75/ $155)$ for the olanzapine group and $38.4 \%(61 / 159)$ for the placebo group, which were not statistically significantly different. Of those who discontinued, $11.0 \%(17 / 155)$ of people in the olanzapine group and $11.3 \%(18 / 159)$ in the placebo group discontinued

\begin{tabular}{|c|c|c|c|}
\hline Characteristic & $\begin{array}{c}\text { Olanzapine } \\
n=155\end{array}$ & $\begin{array}{c}\text { Placebo } \\
n=159\end{array}$ & $P$ \\
\hline Female, $n(\%)$ & 113 (72.9) & $110 \quad(69.2)$ & 0.534 \\
\hline Age, years: mean (s.d.) & $31.8(9.5)$ & $31.8 \quad(9.6)$ & 0.656 \\
\hline White, $n(\%)$ & $136 \quad(87.7)$ & $137 \quad(86.2)$ & 0.872 \\
\hline ZAN-BPD total score, mean (s.d.) & $17.0 \quad(5.2)$ & $17.7 \quad(5.2)$ & 0.156 \\
\hline SCL-90-R Global Severity Index, mean (s.d.) & $1.66(0.8)$ & $1.79(0.7)$ & 0.192 \\
\hline MADRS total score, mean (s.d.) & $12.5 \quad(4.9)$ & $13.2(4.5)$ & 0.133 \\
\hline Current GAF, mean (s.d.) & $54.0(10.1)$ & $53.5(10.3)$ & 0.564 \\
\hline OAS-M Aggression score, mean (s.d.) & $41.2(57.1)$ & $51.0(100.8)$ & 0.234 \\
\hline OAS-M Irritability score, mean (s.d.) & $5.6(1.6)$ & $5.6(1.8)$ & 0.561 \\
\hline OAS-M Suicidality score, mean (s.d.) & $1.1(1.4)$ & $1.2(1.2)$ & 0.412 \\
\hline Sheehan Disability Scale total, mean (s.d.) & $19 \quad(6.0)$ & $20.0(6.4)$ & 0.153 \\
\hline History of major depressive disorder, $n$ (\%) & $58 \quad(37.9)$ & $5 \quad(35.3)$ & 0.639 \\
\hline Unemployed owing to study disease, $n$ (\%) & $33 \quad(21.3)$ & $44 \quad(27.7)$ & NA \\
\hline
\end{tabular}




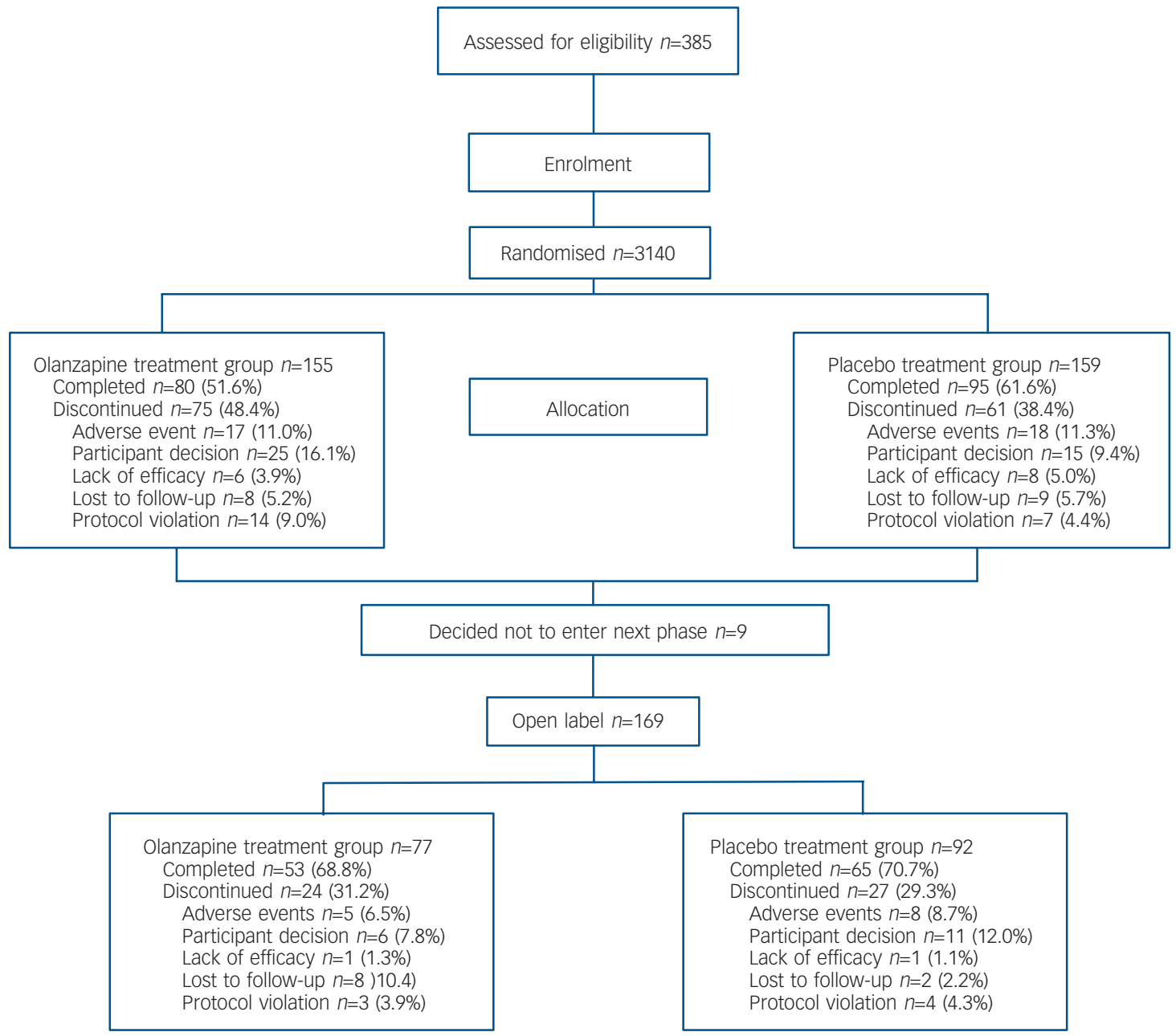

Fig. 1 Patient disposition flow chart, double-blind and open-label extension study periods, all entered patients.

due to an adverse event, while $16.1 \%(25 / 155)$ in the olanzapine group and $9.4 \%(15 / 159)$ in the placebo group discontinued due to participant decision. The mean times to discontinuation were 64.5 days (s.d.=35.6) for olanzapine and 67 days (s.d.=28.7) for placebo. Rates of discontinuation from the study by visit varied from $0-12 \%$, with the highest rates occurring at the 4 - and 6-week time points in both the olanzapine $(12 \%$ and $10.4 \%)$ and placebo (10.3\% and $9.4 \%)$ treatment groups

The mean modal dose of olanzapine during the double-blind period was $7.09 \mathrm{mg} / \mathrm{day}$ (s.d.=5.11). The most common doses of olanzapine were $2.5 \mathrm{mg} /$ day $(n=30,19.7 \%), 5.0 \mathrm{mg} /$ day $(n=31$, $20.4 \%), \quad 7.5 \mathrm{mg} /$ day $(n=28,18.4 \%)$, and $10 \mathrm{mg} /$ day $\quad(n=20$, $13.2 \%)$. Of the 48 participants in the olanzapine group who did not meet the response criteria of $\geqslant 30 \%$ decrease from baseline ZAN-BPD total score at week 4, $36(75 \%)$ were prescribed a dose increase (31 (64.6\%) increased by $2.5 \mathrm{mg} /$ day, 5 (10.4\%) increased by $5 \mathrm{mg}$ /day). At the 6-, 8-, and 10 -week time points, the percentages of participants meeting criteria for a dose increase who were prescribed a dose increase were $70.8 \%, 64.0 \%$ and $50 \%$ respectively.

The incidence of benzodiazepine use did not differ significantly between treatment groups (olanzapine $23.2 \% v$. placebo $27.7 \%$ ), nor did the mean days of benzodiazepine use (olanzapine 25.8 days $v$. placebo 36.4 days; $P=0.077$ ), nor the mean daily dose of benzodiazepines (olanzapine $1.95 \mathrm{mg} \quad v$. placebo $1.55 \mathrm{mg}$; $P=0.337$ ). Only 14 participants were receiving psychotherapy during the study (11 olanzapine $v .3$ placebo).

\section{Efficacy}

Primary outcome measure

Mean ZAN-BPD total scores decreased significantly from baseline to end-point in both olanzapine $(-6.56, P<0.001)$ and placebo $(-6.25, P<0.001)$ treatment groups; however, no significant difference was observed between the treatment groups $(P=0.661$; effect size 0.03 ; $95 \%$ CI -0.20 to -0.25 ).

\section{Secondary outcome measures}

In the visit-wise MMRM analysis of ZAN-BPD total scores, statistically significant separation between treatment groups was observed at the 6- and 8-week time points (Fig. 2). A greater proportion of individuals treated with olanzapine met the $\geqslant 50 \%$ reduction in ZAN-BPD criterion for response relative to placebo, although this difference did not reach statistical significance $(64.7 \%$ v. $53.5 \%, P=0.062)$. When response was defined as a $\geqslant 30 \%$ reduction at any time, response rates were $82.7 \%$ for participants taking olanzapine and $74.2 \%$ for participants taking placebo $(P=0.095)$. Time to reach the $\geqslant 50 \%$ response criterion was statistically significantly shorter for people treated with olanzapine relative to placebo $(P=0.022)$. Mean baseline to end-point decreases in SCL-90-R GSI scores did not differ significantly between treatment groups (olanzapine -0.60 , placebo - 0.56; $P=0.192$ ).

Further analyses of individual items and sub-scales (not corrected for multiple comparisons) identified significantly 


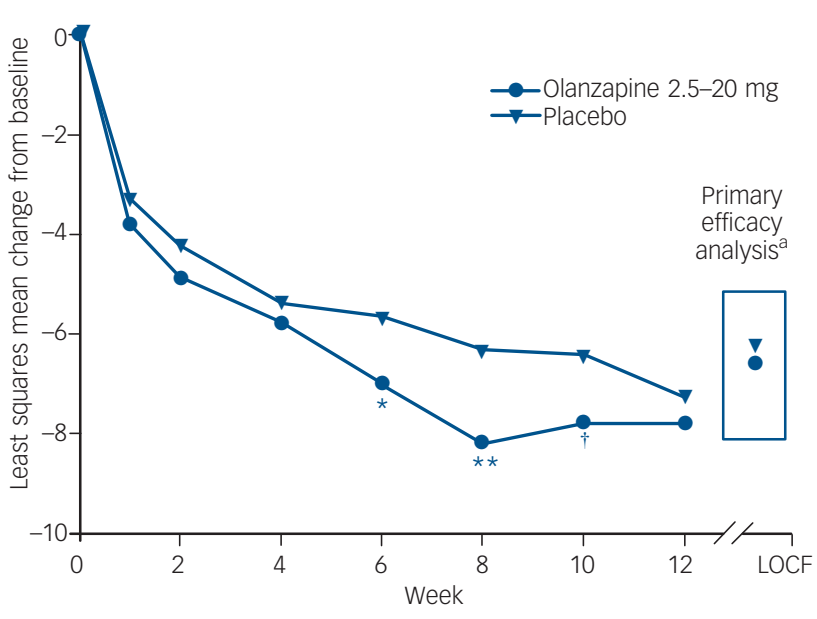

Fig. 2 Mean visit-wise changes in Zanarini Rating Scale for Borderline Personality Disorder (ZAN-BPD) total scores (mixed-effects model repeated measures), and mean baseline to end-point change in ZAN-BPD total score, last observation carried forward (LOCF).

a. Type III sum of squares (ANOVA): model = baseline, investigator, therapy; $P=0.661$

${ }^{\star} P<0.05 ;{ }^{* *} P<0.01 ;{ }^{\dagger} P=0.08$

greater mean (s.e. $(95 \% \mathrm{CI})$ ) baseline to end-point decreases in the Intense Anger item score of the ZAN-BPD for participants taking olanzapine $v$. placebo $(-0.9$, s.e. $=0.09(95 \% \mathrm{CI}-1.08$ to $-0.73)$ v. -0.7 , s.e. $=0.09$ ( $95 \% \mathrm{CI}-0.82$ to -0.47$), P=0.032)$, whereas decreases on the suicidal/self-mutilating behaviour item score were significantly greater for individuals taking placebo $v$. olanzapine $(-0.6$, s.e. $=0.08(95 \% \mathrm{CI}-0.71$ to -0.39$)$ v. -0.3 , s.e. $=0.08$ (95\% CI -0.46 to -0.14$) ; P=0.023)$. Mean LOCF improvements from baseline to end-point were statistically significantly greater for olanzapine- relative to placebo-treated individuals on OAS $-\mathrm{M}$ irritability $(-2.06$, s.e. $=0.18(95 \% \mathrm{CI}-2.41$ to $-1.71) \quad$ v. $\quad-1.51$, s.e. $=0.17 \quad(95 \%$ CI -1.85 to -1.17$)$, $P=0.019)$, Sheehan family life $(-2.05$, s.e. $=0.24$ (95\% CI -2.53 to -1.58$)$ v. -1.39 , s.e. $=0.23(95 \%$ CI -1.85 to -1.93$)$, $P=0.038)$, and SCL-90-R Hostility $(-0.98$, s.e. $=0.08$ (95\% CI $(-1.15$ to -0.82$) \quad$ v. -0.72 , s.e. $=0.08 \quad(95 \%$ CI -0.88 to $-0.56), P=0.019)$ scores. Mean reductions from baseline to LOCF end point in MADRS total score were not significantly different between treatment groups (least squares mean change: -0.11 olanzapine, -0.26 placebo, $P=0.859$ ).

\section{Safety}

Adverse events

Treatment-emergent adverse events were defined as those that first appeared or worsened during double-blind therapy. Among treatment-emergent adverse events reported with a frequency $\geqslant 5 \%$ of either group: somnolence, sedation, increased appetite and weight increase were reported significantly more frequently by people taking olanzapine compared with those treated with placebo (Table 2). During the double-blind study period a total of 15 individuals experienced serious adverse events: six people treated with olanzapine (four reports of suicidal ideation, one report each of aggression, agitation, alcoholism, drug misuse, impulsive behaviour, self-mutilation and self-injurious ideation) and nine people treated with placebo (four reports of suicidal ideation, two reports each of aggression, anxiety, exacerbation of borderline personality disorder symptoms and one report each of depressed mood, fatigue and weight decrease). No deaths occurred during the study.

\section{Weight and vital signs}

Participants in the olanzapine treatment group experienced significantly greater weight gain; mean baseline to end-point change in weight was $2.86 \mathrm{~kg}$ (s.d.=3.02) for the olanzapine group and $-0.35 \mathrm{~kg}($ s.d. $=2.68)$ for the placebo group $(P<0.001)$. The incidence of treatment-emergent weight gain $\geqslant 7 \%$ of baseline was significantly higher for individuals treated with olanzapine relative to those treated with placebo $(34.2 \%$ (51/149) v. $2.6 \%$ (4/155), $P<0.001)$. No significant group differences were observed for blood pressure or pulse.

\section{Metabolic parameters}

Participants treated with olanzapine experienced significantly greater mean increases from baseline to end-point in fasting total cholesterol $(0.17 \mathrm{mmol} / \mathrm{l}(\mathrm{s} . \mathrm{d} .=0.74)$ v. $-0.08 \mathrm{mmol} / \mathrm{l}(\mathrm{s} . \mathrm{d} .=0.60)$; $P=0.003)$ and fasting LDL cholesterol $(0.13 \mathrm{mmol} / \mathrm{l}(\mathrm{s} . \mathrm{d} .=0.65) v$. $-0.08 \mathrm{mmol} / \mathrm{l}$ (s.d. $=0.55$ ); $P=0.007$ ) relative to placebo. Using American Diabetic Association and National Cholesterol Education Program criteria for glucose and lipid parameters respectively, there were no significant differences between treatment groups in the incidence of treatment-emergent abnormal fasting glucose or lipids at any time during treatment.

\section{Prolactin and other laboratory values}

Participants taking olanzapine experienced significantly greater mean elevations from baseline to end-point in prolactin compared

\begin{tabular}{|c|c|c|c|}
\hline Treatment-emergent adverse event & Olanzapine $(n=155), n(\%)$ & Placebo $(n=159), n(\%)$ & $P$ \\
\hline Participants with $\geqslant 1$ treatment-emergent adverse event & $102(65.8)$ & $90(56.6)$ & 0.106 \\
\hline Headache & $23(14.8)$ & $18(11.3)$ & 0.404 \\
\hline Appetite increased & $27(17.4)$ & $12(7.5)$ & 0.010 \\
\hline Weight increased & $27(17.4)$ & $4(2.5)$ & $<0.001$ \\
\hline Fatigue & $16(10.3)$ & $12(7.5)$ & 0.432 \\
\hline Somnolence & $20(12.9)$ & $7(4.4)$ & 0.008 \\
\hline Sedation & $18(11.6)$ & $2(1.3)$ & $<0.001$ \\
\hline Nausea & $7(4.5)$ & $12(7.5)$ & 0.345 \\
\hline Dry mouth & $11(7.1)$ & $6(3.8)$ & 0.220 \\
\hline Anxiety & $7(4.5)$ & $8(5.0)$ & 1.00 \\
\hline Insomnia & $4(2.6)$ & $10(6.3)$ & 0.170 \\
\hline Suicidal ideation & $9(5.8)$ & $4(2.5)$ & 0.166 \\
\hline
\end{tabular}


with those taking placebo $(7.75 \mathrm{~g} / 1 \quad$ (s.d.=27.96) $\quad v . \quad 0.65 \mathrm{~g} / \mathrm{l}$ (s.d.=14.82); $P=0.006$ ). The incidence of treatment-emergent abnormal high levels of prolactin at end-point was statistically significantly higher for individuals treated with olanzapine relative to those treated with placebo $(27.5 \%(30 / 109)$ v. $12.1 \%(14 / 116)$, $P=0.004)$. Those taking olanzapine also experienced statistically significantly greater mean elevations from baseline to end-point in hepatic enzymes aspartate transaminase (AST) $(2.32 \mathrm{u} / \mathrm{l}$ (s.d.=9.12) $v . \quad-1.07 \mathrm{u} / \mathrm{l}($ s.d. $=6.91) ; P=0.001)$ and alanine transaminase (ALT) $(5.33 \mathrm{u} / \mathrm{l}($ s.d.=21.14) v. $-1.94 \mathrm{u} / \mathrm{l} \quad($ s.d.=11.95); $P=0.001)$ relative to those treated with placebo. The incidence of treatment-emergent abnormal high levels of hepatic enzymes did not differ significantly between treatment groups. Statistically significant differences between the olanzapine and placebo treatment groups were observed for mean baseline to end-point changes in total bilirubin $(-0.87 \mathrm{~mol} / \mathrm{l}(\mathrm{s} . \mathrm{d} .=3.69) v .0 .11 \mathrm{~mol} / \mathrm{l}$ (s.d.=3.06); $P=0.013)$ and direct bilirubin $(-0.27 \mathrm{~mol} / \mathrm{l}$ (s.d. $=0.94)$ v. $0.03 \mathrm{~mol} / \mathrm{l}$ (s.d.=0.74); $P=0.005$ ).

\section{Electrocardiogram}

There were no significant differences between treatment groups on any of the ECG measures, and no significant differences were observed between treatment groups in the analysis of potentially clinically significant changes in ECG. In an analysis of potentially clinically significant change in QTc intervals using additional criteria, there were no significant differences between treatment groups.

\section{Extrapyramidal symptoms}

No significant differences were observed between treatment groups with respect to EPS as assessed by mean changes from baseline to end-point in scores on the AIMS, Simpson-Angus, or Barnes Akathisia Rating scales.

\section{Discussion}

This study represents one of two large, placebo-controlled trials of olanzapine in the treatment of borderline personality disorders and, as such, breaks new ground in pharmacotherapy research with regard to dosing and assessment strategies in a controlled trial, inclusion/exclusion criteria for this heterogeneous disorder and the psychosocial aspects of individuals' experience during the study.

In this randomised, controlled trial of borderline personality disorder, participants improved significantly over the 12 weeks of study; however, the olanzapine- and placebo-treated groups were not significantly different in the main assessment of efficacy - symptom reduction on the ZAN-BPD. Baseline scores were indicative of moderate symptom severity and were consistent with those of an out-patient population. After 12 weeks of treatment, mean scores in both treatment groups were indicative of mild symptom severity. Interestingly, there was a nearly statistically significant difference between olanzapine and placebo in the number of people who were considered responders $(\geqslant 50 \%$ decrease from baseline ZAN-BPD total score $)(P=0.06)$ and a significant separation in the time of response $(P=0.02)$, with the olanzapine group achieving response more quickly.

As the lack of significant separation between treatment groups on the ZAN-BPD, SCL-90-R GSI, and OAS-M scores differs from the results of previous studies, ${ }^{6,10-12,30}$ this difference deserves exploration. It should also be noted that the present results differ from those of a large placebo-controlled olanzapine dose comparison study that was conducted simultaneously. ${ }^{13}$ Possible causes for a lack of advantage of olanzapine compared with placebo in the present study may have been secondary to participant sample, underdosing or response of individuals to the support of the investigative team. Furthermore, large trials in psychiatry are not always in agreement ${ }^{31,32}$ and there have been previous studies in borderline personality disorder in which active medication was not statistically superior to placebo. ${ }^{27}$

Characteristics of the participant sample may have led to a lack of difference between individuals assigned to olanzapine $v$. placebo. However, examination of response between men and women did not reveal any differences, and there were similar proportions of men and women in each group. Subgroup analyses were also conducted to assess for possible interaction between outcome and age (by comparing people above or below the age of 26) or origin. Neither of these subgroup analyses were statistically significant.

One hypothesis regarding the lack of differences between treatment groups in the present study is that participants were not titrated to a high enough dose of olanzapine. Because this study was not designed as a dose comparison study, it is not possible to draw conclusions here regarding the relationship between dose and efficacy. However, it should be noted that $50 \%$ of the participants received $5 \mathrm{mg} /$ day or less as their most common daily dose $(20.4 \%$ received $5 \mathrm{mg}, 19.7 \%$ received $2.5 \mathrm{mg}$, and $9.9 \%$ received $0 \mathrm{mg}$ as their most frequent dose). It should also be noted that these doses represent the dose taken and not necessarily the dose prescribed. Thus, the finding that $9.9 \%$ of individuals reported taking $0 \mathrm{mg}$ as their most frequent dose indicates that, although those participants received olanzapine at times during the study, a considerable portion of the time was spent without the drug. This suggests that despite the attempt to ensure adequate dosing via the forced titration scheme, underdosing cannot be ruled out as a possible explanation for the current findings. Future study designs for this population might do well to incorporate a more rigorous treatment response criterion (e.g. 50\% response) and/or an earlier time point for implementing upward dose titration. In the present study, a majority of participants had already achieved the $\geqslant 30 \%$ response criterion by the 4 -week time point at which assessments for upward dose titration were required. An analysis of the pattern of dose titrations revealed that although the majority of people for whom titration was indicated were prescribed a dose increase, the increases were modest (most commonly $2.5 \mathrm{mg}$ ). Furthermore, the proportions of individuals who met criteria for a dose increase, but were not prescribed a higher dose, increased as the trial progressed. These findings suggest that clinical investigators may have been cautious about increasing the dose of olanzapine to a level that is necessary for an adequate trial. Another consideration for future study designs would be to mask participants to dose changes. Although participants were not aware of their treatment assignment, they were responsible for taking the prescribed number of tablets from their dose packs to achieve the correct dose.

As for the finding that both treatment groups showed significant symptom reduction, one influential factor may have been the structure and attention the participants received in this 12-week trial. Other studies involving people with borderline personality disorder have also reported significant decreases in functional scores for both the medication and placebo groups, ${ }^{33}$ and in a small, placebo-controlled, pilot study, Schulz et al (details available from the author) reported that both risperidone- and placebo-treated participants had significant reductions in symptom scores. Nevertheless, equivalent symptom reductions in groups are not always seen in medication studies when increased attention is given to people with borderline 
personality disorder during a trial. For instance, Soler et al have shown an advantage for olanzapine compared with placebo in a trial in which all individuals also received dialectical behaviour therapy. ${ }^{10}$

In the present study, the overall safety findings with respect to treatment-emergent adverse events, weight gain, laboratory values and metabolic parameters were consistent with those of previous studies of olanzapine in adult populations. ${ }^{34,35}$ The most notable findings were the higher incidence of clinically significant weight gain and treatment-emergent elevated prolactin in the olanzapine group relative to the placebo group. Also noteworthy was the absence of significant treatment-emergent EPS. Suicidal ideation, which is commonly observed in those with borderline personality disorder, ${ }^{4}$ was reported in both olanzapine and placebo groups; however, no deaths occurred during the study.

In addition to the studies of atypical antipsychotics mentioned previously, numerous medications representing different classes of compounds have been evaluated for efficacy in the treatment of people with borderline personality disorder. Some studies ${ }^{33,36}$ but not others, ${ }^{37}$ have shown an advantage of traditional antipsychotic medications over placebo. Selective serotonin reuptake inhibitor antidepressant drugs have also shown effectiveness in case series ${ }^{38,39}$ and double-blind trials. ${ }^{40,41}$ Similarly, a serotonin-norepinephrine reuptake inhibitor, venlafaxine, has been reported to be superior to placebo in this population. ${ }^{42}$ Mood-stabilising anticonvulsants have also been shown to be useful $^{14,43}$ in controlled trials. Interestingly, not all classes of compounds have demonstrated benefit, as tricyclic antidepressants appear to increase agitation in borderline personality disorder ${ }^{36}$ and alprazolam, a benzodiazepine, can be followed by disinhibition. ${ }^{37}$

In conclusion, our paper describes one of two concurrently conducted trials of olanzapine $v$. placebo conducted at multiple, international sites. In this study, which tested a variable dosing strategy, olanzapine was not statistically different from placebo on the primary efficacy measure; however, participants who received olanzapine achieved clinical response more quickly than did those on placebo. Although the results of the study were not positive on the primary measure, the study demonstrates the feasibility of large, multicentre investigations in this largely ignored population. Furthermore, this rich data-set may provide additional information regarding disease characteristics and treatment outcomes.

\footnotetext{
S. Charles Schulz, MD, Department of Psychiatry, University of Minnesota Medical school, Minneapolis, MN, USA; Mary C. Zanarini, EdD, McLean Hospital, Harvard Medical School, Boston, MA, USA; Anthony Bateman, MA, FRCPsych, Halliwick Unit, Medical School, Boston, MA, USA; Anthony Bateman, MA, FRCPSych, Halliwick Un,
St Ann's Hospital, Barnet, Enfield and Haringey Mental Health Trust, London, UK; Martin Bohus, MD, Department of Psychosomatic Medicine, Central Institute of Mental Health, University of Heidelberg, Germany; Holland C. Detke, PhD, Quynh Trzaskoma, MA, Yoko Tanaka, PhD, Daniel Lin, PhD, Lilly Research Laboratories, Indianapolis, IN, USA; Walter Deberdt, MD, Lilly Research Laboratories, Belgium; Sara Corya, MD, Lilly Research Laboratories, Indianapolis, IN, USA

Correspondence: Dr S. Charles Schulz, Department of Psychiatry, Medical School, F282/2A West, 2450 Riverside Avenue, Minneapolis, MN 55454, USA. Email: scs@umn.edu

First received 23 Mar 2007, final revision 17 Apr 2008, accepted 29 Apr 2008
}

\section{Acknowledgements}

The authors thank the F1D-MC-HGKL Study Group investigators for their significant contributions to site management and data collection. The authors also thank Dr Ludmilla Kryzhanovskaya for medical review, and Cheryl R. Brown and the F1D-MC-HGKL clinical Kryzhanovskaya for medical review, and Cheryl R. Brown and the F1D-MC-HGKL Clinical
operations team for project management. This work was sponsored by Eli Lilly and company. Trial Registry: NCT00091650, http://Www.clinicaltrials.gov/.

\section{References}

1 Swartz M, Blazer D, George L, Winfield I. Estimating the prevalence of borderline personality disorder in the community. J Personal Disord 1990; 4 257-72.

2 Torgersen S, Kringlen E, Cramer V. The prevalence of personality disorders in a community sample. Arch Gen Psychiatry 2001; 58: 590-6.

3 Skodol AE, Gunderson JG, McGlashan TH, Dyck IR, Stout RL, Bender DS, Grilo CM, Shea MT, Zanarini MC, Morey LC, Sanislow CA, Oldham JM. Functional impairment in patients with schizotypal, borderline, avoidant, or obsessivecompulsive personality disorder. Am J Psychiatry 2002; 159: 276-83.

4 Stone MH. Long-term outcome in personality disorders. Br J Psychiatry 1993; 162: 299-313.

5 Benedetti F, Sforzini L, Colombo C, Maffei C, Smeraldi E. Low-dose clozapine in acute and continuation treatment of severe borderline personality disorder. J Clin Psychiatry 1998; 59: 103-7.

6 Bogenschutz MP, George NH. Olanzapine versus placebo in the treatment of borderline personality disorder. J Clin Psychiatry 2004; 65: 104-9.

7 Frankenburg FR, Zanarini MC. Clozapine treatment of borderline patients: a preliminary study. Compr Psychiatry 1993; 34: 402-5.

8 Nickel MK, Muehlbacher M, Nickel C, Kettler C, Pedrosa GF, Bachler E, Buschmann W, Rother N, Fartacck R, Egger C, Anvar J, Rother WK, Loew TH, Kaplan P. Aripiprazole in the treatment of patients with borderline personality disorder: a double-blind, placebo-controlled study. Am J Psychiatry 2006; 163: 833-8.

9 Rocca P, Marchiaro L, Cocuzza E, Bogetto F. Treatment of borderline personality disorder with risperidone. J Clin Psychiatry 2002; 63: 241-4.

10 Soler J, Pascual JC, Campins J, Barrachina J, Puigdemont D, Alvarez E, Perez, V. Double-blind, placebo-controlled study of dialectical behavior therapy plus olanzapine for borderline personality disorder. Am J Psychiatry 2005; 162: 1221-4.

11 Zanarini MC, Frankenburg FR. Olanzapine treatment of female borderline personality disorder patients: a double-blind, placebo-controlled pilot study. J Clin Psychiatry 2001; 62: 849-54.

12 Zanarini MC, Frankenburg FR, Parachini EA. A preliminary, randomized trial of fluoxetine, olanzapine, and the olanzapine-fluoxetine combination in women with borderline personality disorder. J Clin Psychiatry 2004; 65: 903-7.

13 Zanarini MC, Schulz SC, Detke HC, Tanaka Y, Zhao F, Lin D, DeBerdt W, Corya S. A dose comparison of olanzapine for the treatment of borderline personality disorder: a 12-week randomized, double-blind, placebo-controlled study. Int J Neuropsychopharmacol 2006; 9 (suppl 1): s191.

14 Frankenburg FR, Zanarini MC. Divalproex sodium treatment of women with borderline personality disorder and bipolar II disorder: a double-blind placebo-controlled pilot study. J Clin Psychiatry 2002; 63: 442-6.

15 Soloff PH, Lynch KG, Kelly TM, Malone KM, Mann JJ. Characteristics of suicide attempts of patients with major depressive episode and borderline personality disorder: a comparative study. Am J Psychiatry 2000; 157: 601-8.

16 Zanarini MC, Vujanovic AA, Parachini EA, Boulanger JL, Frankenburg FR, Hennen J. Zanarini Rating Scale for Borderline Personality Disorder (ZAN-BPD): a continuous measure of DSM-IV borderline psychopathology. J Personal Disord 2003; 17: 233-42.

17 American Psychiatric Association. Diagnostic and Statistical Manual of Mental Disorders (4th edn) (DSM-IV). APA, 1994.

18 First MB, Gibbon M, Spitzer RL, Williams JBW. Structured Clinical Interview for DSM-IV-TR Axis I Disorders, Research Version, Patient Edition (SCID-I/P). Biometrics Research, New York State Psychiatric Institute, 2001.

19 Derogatis LR, Savitz KL. The SCL-90-R, brief symptom inventory and matching clinical rating scales. In The Use of Psychological Testing for Treatment Planning and Outcome Assessment (ed. ME Maruish): 41-80. Lawrence Erlbaum Associates, 1999.

20 American Psychiatric Association. Global Assessment of Functioning (GAF) Scale. In Diagnostic and Statistical Manual of Mental Disorders (4th edn, text revision) (DSM-IV-TR): 32-4. APA, 2000.

21 Sheehan DV, Harnett-Sheehan K, Raj BA. The measurement of disability. Int Clin Psychopharmacol 1996; 11 (suppl 3): 89-95.

22 Coccaro EF, Harvey PD, Kupsaw-Lawrence E, Herbert JL, Bernstein DP Development of neuropharmacologically based behavioral assessments of impulsive aggressive behavior. J Neuropsychiatry Clin Neurosci 1991; 3: s44-S51.

23 Montgomery SA, Åsberg M. A new depression scale designed to be sensitive to change. Br J Psychiatry 1979; 134: 382-9. 
24 Davidson J, Turnbull CD, Strickland R, Miller R, Graves K. The MontgomeryÅsberg depression rating scale: reliability and validity. Acta Psychiatr Scand 1986; 73: $544-8$.

25 National Cholesterol Education Program. Third report of the National Cholesterol Education Program (NCEP) Expert Panel on detection, evaluation, and treatment of high blood cholesterol in adults (Adult Treatment Panel III) final report. Circulation 2002; 106: 3143-421.

26 American Diabetic Assocation. Standards of medical care in diabetes. Diabetes Care 2004; 27 (suppl 1): s15-35.

27 Simpson EB, Yen S, Costello E, Rosen K, Begin A, Pistorello J, Pearlstein T. Combined dialectical behavior therapy and fluoxetine in the treatment of borderline personality disorder. J Clin Psychiatry 2004; 65: 379-85.

28 Barnes TR. A rating scale for drug-induced akathisia. Br J Psychiatry 1989; 154: $672-6$.

29 Guy W. ECDEU Assessment Manual for Psychopharmacology, Revised. US Department of Health, Education and Welfare, 1976.

30 Schulz SC, Camlin KL, Berry SA, Jesberger JA. Olanzapine safety and efficacy in patients with borderline personality disorder and comorbid dysthymia. Biol Psychiatry 1999; 46: 1429-35.

31 Khan A, Kolts RL, Rapaport MH, Krishnan KR, Brodhead AE, Browns WA. Magnitude of placebo response and drug-placebo differences across psychiatric disorders. Psychol Med 2005; 5: 743-9.

32 Walsh BT, Seidman SN, Sysko R, Gould M. Placebo response in studies of major depression: variable, substantial, and growing. JAMA 2002; 287 $1840-7$.

33 Goldberg SC, Schulz SC, Schulz PM, Resnick RJ, Hamer RM, Friedel RO. Borderline and schizotypal personality disorders treated with low-dose thiothixene vs placebo. Arch Gen Psychiatry 1986; 43: 680-6.

34 Tohen M, Sanger TM, McElroy SL, Tollefson GD, Chengappa KN, Daniel DG, Petty F, Centorrino F, Wang R, Grundy SL, Greaney MG, Jacobs TG, David SR,
Toma V. Olanzapine versus placebo in the treatment of acute mania. Am J Psychiatry 1999; 156: 702-9.

35 Tohen $\mathrm{M}$, Jacobs TG, Grundy SL, Banov MC, McElroy SL, Janicak PG, Sanger T, Risser R, Zhang F, Toma V, Francis J, Tollefson GD, Breier A. Efficacy of olanzapine in acute bipolar mania: a double-blind, placebo-controlled study. Arch Gen Psychiatry 2000; 57: 841-9.

36 Soloff PH, George A, Nathan RS, Schulz PM, Ulrich RF, Perel JM. Progress in pharmacotherapy of borderline disorders. A double-blind study of amitriptyline, haloperidol, and placebo. Arch Gen Psychiatry 1986; 43: 691-7.

37 Cowdry RW, Gardner DL. Pharmacotherapy of borderline personality disorder. Alprazolam, carbamazepine, trifluoperazine, and tranylcypromine. Arch Gen Psychiatry 1988; 45: 111-9.

38 Cornelius JR, Soloff PH, Perel JM, Ulrich RF. A preliminary trial of fluoxetine in refractory borderline patients. J Clin Psychopharmacol 1991; 11: 116-20.

39 Markovitz PJ, Calabrese JR, Schulz SC, Meltzer HY. Fluoxetine in the treatment of borderline and schizotypal personality disorders. Am J Psychiatry 1991; 148: 1064-7.

40 Coccaro EF, Kavoussi RJ. Fluoxetine and impulsive aggressive behavior in personality-disordered subjects. Arch Gen Psychiatry 1997; 54: 1081-8.

41 Salzman C, Wolfson AN, Schatzberg A, Looper J, Henke R, Albanese M, Schwartz J, Miyawaki, E. Effect of fluoxetine on anger in symptomatic volunteers with borderline personality disorder. J Clin Psychopharmacol 1995; 15: 23-9.

42 Markovitz PJ, Wagner SC. Venlafaxine in the treatment of borderline personality disorder. Psychopharmacol Bull 1995; 31: 773-7.

43 Hollander E, Tracy KA, Swann AC, Coccaro EF, McElroy SL, Wozniak P, Sommerville $\mathrm{KW}$, Nemeroff $\mathrm{CB}$. Divalproex in the treatment of impulsive aggression: efficacy in cluster B personality disorders. Neuropsychopharmacol 2003; 28: 1186-97. 